\title{
BRD4 jump-starts transcription after mitotic silencing
}

\author{
Philipp Voigt and Danny Reinberg*
}

\begin{abstract}
The chromatin adapter BRD4 may be crucial for transmitting epigenetic information by acting as a histone acetylation-dependent gene bookmark and accelerating post-mitotic transcriptional reactivation.
\end{abstract}

Mitosis in all eukaryotic cells is accompanied by a nearcomplete shutdown of transcription due to highly compacted chromatin and dissociation of transcription factors, chromatin remodelers and components of the basal transcription machinery from mitotic chromosomes. Upon exit from mitosis, transcription resumes and gene expression patterns must be restored faithfully in order to maintain cellular identity. However, as most components of the transcription and chromatin-modifying machinery are either displaced or degraded when entering mitosis, timely and accurate reactivation of the genes necessary for cell cycle progression and maintenance of cell fate poses a significant challenge. How cells remember the transcriptional program that defines their lineage and identity during mitosis remains a key question in chromatin biology.

The emerging concept of gene bookmarking unifies potential mechanisms by which genes are marked for post-mitotic re-activation or re-silencing so that their transcriptional state is accurately passed on to progeny cells [1]. In a recent elegant study, Zhao et al. [2] employed real-time quantitative fluorescence microscopy to assess the kinetics of transcriptional reactivation after mitosis. Their findings support a bookmarking mechanism centered on bromodomain protein 4 (BRD4), a member of the bromodomain and extraterminal (BET) family of proteins, and a promising target for cancer therapeutics.

\section{Real-time imaging of RNA polymerase II shows a return to bookmarked genes}

To follow the kinetics of post-mitotic transcriptional restart in real time in living cells, Zhao et al. made use of

*Correspondence: danny.reinberg@nyumc.org

Howard Hughes Medical Institute, New York University School of Medicine,

Department of Biochemistry, 522 First Avenue, New York, NY 10016, USA their previously developed inducible reporter transgene array, whose DNA locus and nascent transcripts can be simultaneously visualized by fluorescence microscopy [3]. Upon induction with a Tet-On system, fluorescently labeled RNA polymerase II (Pol II) was gradually and slowly recruited to the reporter locus, reaching a plateau after about $3.5 \mathrm{~h}$ [2]. Nascent transcripts were detected with similar kinetics.

Induced cells were monitored as they left interphase and progressed through mitosis. Pol II disappeared from the locus at the onset of mitosis, and no transcripts were detectable throughout mitosis, as expected from the general mitotic shutdown of transcription. After mitosis, Pol II was re-recruited to the reporter locus with much faster kinetics than those of the initial induction, a behavior that was reflected in the kinetics of the nascent transcripts. The locus was thus not only marked for postmitotic reactivation, it also gained a kinetic edge in passage through mitosis that led both to a faster onset and steeper rise of recruitment.

Importantly, the Tet-On activator was lost from chromatin at the onset of mitosis, precluding the possibility that the bookmarking and enhanced re-activation kinetics were due to its continued association with the locus. This observation suggests the presence of an activator-independent memory system for gene bookmarking.

\section{Hunting for the molecular bookmark}

By definition, a gene bookmark must remain associated with its target genes during mitosis, and this association must be transmitted to the daughter cells after cytokinesis. Several transcription factors and regulatory proteins fit this profile as they resist displacement and remain bound to a number of target sites on mitotic chromosomes. More recently, additional signals have been recognized as potential bookmarks. These comprise epigenetic marks such as DNA methylation, histone variants and histone modifications, as well as the enzymes that place these marks and the factors that recognize them [1].

To elucidate the nature of the bookmark in this setting, the authors turned to analyzing the presence of activating histone marks at the locus by chromatin immunoprecipitation (ChIP). Concomitant with induction of the 
reporter gene in interphase, the promoter region gained mostly acetylation at lysine 5 of histone $\mathrm{H} 4$ (H4K5ac), with little changes in other activating marks. If this signal bookmarks the reporter for reactivation, it has to persist through mitosis. Indeed, H4K5ac remained elevated at the promoter in nocodazole-arrested metaphase cells. In pinpointing which component of the transcriptional machinery might decipher this potential epigenetic bookmark, the authors focused on BRD4, based on its established acetyl-lysine binding properties, transcriptional coactivator function, and its retention on chromatin at certain loci throughout mitosis. In immunofluorescence experiments, BRD4 was found to be associated with the reporter locus in about $50 \%$ of induced cells during mitosis. Upon exit from mitosis, BRD4 levels at the reporter increased even further in all previously induced cells, suggesting that BRD4 indeed plays a crucial role in bookmarking that locus.

In addition to the data presented by Zhao et al., BRD4 has several features rendering it a promising candidate for an efficient bookmark: it is a member of several transcription complexes and acts predominantly as a transcriptional coactivator (through its interaction with positive transcription elongation factor $b$ (pTEFb) [4]), and its tandem bromodomains bind to acetylated histone tails, preferentially those of $\mathrm{H} 4$ acetylated at two or more lysines. Most tellingly, one of the defining characteristics of BRD4 is its retention on mitotic chromosomes in a range of cell types. This is perhaps surprising given the chromatin binding specificity of BRD4, since overall histone acetylation levels are markedly reduced on mitotic chromosomes; however, certain transcriptional start sites retain acetylation throughout mitosis. Intriguingly, BRD4 has been shown to localize to these regions during mitosis [5]. In addition, binding of BRD4 to these sites rapidly increased at the onset of telophase, concurrent with enhanced acetylation [5]. Both phenomena are likely to be mediated by interaction between BRD bromodomains and acetyl-lysines, suggesting an active role for BRD proteins and acetylated lysines in a bookmarking system.

\section{Is chromatin decompaction key to the bookmarking mechanism of BRD4?}

Interestingly, Zhao et al. found that recruitment of BRD4 preceded that of Pol II in post-mitotic cells, whereas the opposite was observed for interphase induction. In interphase, BRD4 recruitment is subsequent to Pol II recruitment but parallels that of the pTEFb complex subunit Cdk9, which phosphorylates serine 2 within the carboxy-terminal repeats of Pol II to promote elongation. These findings indicate that BRD4 was recruited as part of a functional transcription elongation complex during interphase induction. However, after mitosis, BRD4 binding preceded both Pol II and Cdk9 recruitment. This distinction in kinetics indicates that BRD4 is likely to function outside of the pTEFb transcription elongation complex in accelerating post-mitotic gene activation.

The observation that the transgene locus, visualized by the fluorescent lacI fusion protein, takes up a larger volume in the nucleus when bound by BRD4 may point to BRD4 exerting its bookmark function by engaging $\mathrm{H} 4 \mathrm{~K} 5 \mathrm{ac}$ at the locus and establishing a less compact and more accessible chromatin environment, facilitating reactivation of the locus. The first bromodomain of BRD4 is both necessary and sufficient for chromatin decompaction, and both full-length BRD4 and the isolated bromodomain can establish a decondensed structure at the reporter when artificially recruited as lacI fusions. Further studies are necessary to determine whether decompaction is the predominant mode of action for BRD4 in bookmarking; other mechanisms, such as the efficient recruitment of transcription complexes, are likely to exist in parallel with, or alternatively to, decompaction. Moreover, it cannot be ruled out that the highly repetitive architecture of the reporter, featuring arrays of hundreds of binding sites, may distort kinetics and chromatin environments. Although technically challenging, it would be fascinating to observe in real time postmitotic reactivation of loci that more closely resemble actual gene structures. Despite those technical limitations, the work of Zhao et al. provides us with novel insights into the kinetics of BRD4 recruitment and its role in gene bookmarking.

\section{All BETs are off: BET inhibitors, gene bookmarking and cancer}

Recently, bromodomain proteins, and BRD4 in particular, have received a significant degree of attention because of the discovery of two small molecules capable of disrupting the interaction between BRD bromodomains and acetylated histones. These compounds are designed to mimic acetylated histone tails in order to efficiently compete for binding to the BET but not other bromodomains.

One of the compounds, I-BET (GSK525762A), binds BET proteins with an affinity of about $55 \mathrm{nM}$ and was initially shown to have anti-inflammatory potential through interference with inflammatory gene expression [6]. However, the major potential for these compounds lies in targeting cancer because of the function of BRD4 in mitotic progression by marking genes for timely activation at the end of mitosis and in early G1 [5] and, through its association with pTEFb, in enhancing the transcript levels of c-myc and other oncogenes [4]. The amino-terminal half of BRD4, including the bromodomains, is also found as a fusion with NUT (NUclear protein in Testis) as a result of a chromosomal translocation associated with squamous carcinoma [4]. 
JQ1, the second prototypical BET domain inhibitor, has an affinity comparable to I-BET, and likewise displaces acetylated histone peptides from the BRD4 bromodomains and from fluorescently tagged versions of both BRD4 and the BRD4-NUT fusion from chromatin in cells [7]. Proliferation of cells derived from BRD4-NUTdependent midline carcinoma was diminished upon JQ1 treatment, leading to tumor regression and prolonged survival in mouse models [7]. Several strands of evidence, from a number of patient-derived cancer cell lines and xenograft models, have further shown that JQ1 has potent antitumor efficacy on $m y c$-dependent cancer cells, and it has been proposed that this is due to reduced expression of c-myc and its downstream transcriptional programs $[8,9]$. An elegant, recent study from the Kouzarides laboratory uncovered interactions of BET proteins with mixed lineage leukemia (MLL) fusion proteins as part of the super elongation complex and the polymerase-associated factor (PAF) complex [10]. BET inhibitors had strong antiproliferative efficacy against cell lines harboring MLL fusions and had profound benefits in mouse models of MLL [10], expanding the therapeutic spectrum of BET inhibitors from midline carcinoma and c-myc-dependent cancers to MLL fusion-driven cancers.

The finding by Zhao et al. that BRD4 functions as a gene expression bookmark prompts the question of whether the mode of action of BET inhibitors extends to the disruption of gene bookmarking in addition to the impairment of transcriptional elongation. Zhao et al. addressed this question by examining the impact of JQ1 on the output of their assay, finding that JQ1 treatment generally increased the mobility of BRD4 at actively transcribing reporter cassettes [2]; this is in agreement with previous studies [7] and indicative of a weakened interaction with chromatin. Notably, post-mitotic reactivation was markedly slowed down in the presence of JQ1, whereas interphase induction kinetics remained unaffected [2]. These results are consistent with a pronounced impact of JQ1 (and likely other BET inhibitors) on gene bookmarking.

Abbreviations

BET, bromodomain and extraterminal; BRD4, bromodomain protein 4; $\mathrm{H} 4 \mathrm{~K} 5 \mathrm{ac}$, histone $\mathrm{H} 4$ acetylated at lysine 5: MLL, mixed lineage leukemia; NUT, nuclear protein in testis; POL II, RNA polymerase II; pTEFb, positive transcription elongation factor $b$
Competing interests

The authors declare that they have no competing interests.

\section{Acknowledgements}

PV is supported by a fellowship from the Deutsche Akademie der Naturforscher Leopoldina (LPDS 2009-5). We thank Lynne Vales, David Beck, Emilio Lecona and Roberto Bonasio for helpful discussions and critical reading of the manuscript.

Published: 29 November 2011

\section{References}

1. Zaidi SK, Young DW, Montecino MA, Lian JB, van Wijnen AJ, Stein JL, Stein GS: Mitotic bookmarking of genes: a novel dimension to epigenetic control. Nat Rev Genet 2010, 11:583-589.

2. Zhao R, Nakamura T, Fu Y, Lazar Z, Spector DL: Gene bookmarking accelerates the kinetics of post-mitotic transcriptional re-activation. Nat Cell Biol 2011, 13:1295-1304.

3. Janicki SM, Tsukamoto T, Salghetti SE, Tansey WP, Sachidanandam R, Prasanth KV, Ried T, Shav-Tal Y, Bertrand E, Singer RH, Spector DL: From silencing to gene expression: real-time analysis in single cells. Cell 2004, 116:683-698.

4. Wu S-Y, Chiang C-M: The double bromodomain-containing chromatin adaptor Brd4 and transcriptional regulation. J Bio/ Chem 2007, 282:13141-13145.

5. Dey A, Nishiyama A, Karpova T, McNally J, Ozato K: Brd4 marks select genes on mitotic chromatin and directs postmitotic transcription. Mol Biol Cell 2009, 20:4899-4909.

6. Nicodeme E, Jeffrey KL, Schaefer U, Beinke S, Dewell S, Chung C-W, Chandwani R, Marazzi I, Wilson P, Coste H, White J, Kirilovsky J, Rice CM, Lora JM, Prinjha RK, Lee K, Tarakhovsky A: Suppression of inflammation by a synthetic histone mimic. Nature 2010, 468:1119-1123.

7. Filippakopoulos P, Qi J, Picaud S, Shen Y, Smith WB, Fedorov O, Morse EM, Keates T, Hickman TT, Felletar I, Philpott M, Munro S, McKeown MR, Wang Y, Christie AL, West N, Cameron MJ, Schwartz B, Heightman TD, La Thangue N, French CA, Wiest O, Kung AL, Knapp S, Bradner JE: Selective inhibition of BET bromodomains. Nature 2010, 468:1067-1073.

8. Mertz JA, Conery AR, Bryant BM, Sandy P, Balasubramanian S, Mele DA, Bergeron L, Sims RJ: Targeting MYC dependence in cancer by inhibiting BET bromodomains. Proc Natl Acad Sci U S A 2011, 108:16669-16674.

9. Delmore JE, Issa GC, Lemieux ME, Rahl PB, Shi J, Jacobs HM, Kastritis E, Gilpatrick T, Paranal RM, Qi J, Chesi M, Schinzel AC, McKeown MR, Heffernan TP, Vakoc CR, Bergsagel PL, Ghobrial IM, Richardson PG, Young RA, Hahn WC, Anderson KC, Kung AL, Bradner JE, Mitsiades CS: BET Bromodomain inhibition as a therapeutic strategy to target c-Myc. Cell 2011, 146:904-917.

10. Dawson MA, Prinjha RK, Dittman A, Giotopoulos G, Bantscheff M, Chan W-I, Robson SC, Chung C-W, Hopf C, Savitski MM, Huthmacher C, Gudgin E, Lugo D, Beinke S, Chapman TD, Roberts EJ, Soden PE, Auger KR, Mirguet O, Doehner K, Delwel R, Burnett AK, Jeffrey P, Drewes G, Lee K, Huntly BJ, Kouzarides T: Inhibition of BET recruitment to chromatin as an effective treatment for MLL-fusion leukaemia. Nature 2011, 478:529-533.

doi:10.1186/gb-2011-12-11-133

Cite this article as: Voigt P, Reinberg D: BRD4 jump-starts transcription after mitotic silencing. Genome Biology 2011, 12:133. 